\title{
DOA Estimation Method in Multipath Environment for Passive Bistatic Radar
}

\author{
Panhe Hu $\mathbb{D}^{\mathrm{D}}{ }^{1}$ Qinglong Bao, ${ }^{1}$ and Zengping Chen ${ }^{1,2}$ \\ ${ }^{1}$ National Key Laboratory of Science and Technology on ATR, National University of Defense Technology, Changsha 410073, China \\ ${ }^{2}$ College of Information Engineering, Shenzhen University, Shenzhen 518060, China
}

Correspondence should be addressed to Panhe Hu; hupanhe2009@163.com

Received 24 January 2019; Revised 6 May 2019; Accepted 13 May 2019; Published 20 June 2019

Academic Editor: Ana Alejos

Copyright (C) 2019 Panhe Hu et al. This is an open access article distributed under the Creative Commons Attribution License, which permits unrestricted use, distribution, and reproduction in any medium, provided the original work is properly cited.

\begin{abstract}
Direction-of-arrival (DOA) estimation in multipath environment is an important issue for passive bistatic radar (PBR) using frequency agile phased array VHF radar as illuminator of opportunity. Under such scenario, the main focus of this paper is to cope with the closely spaced uncorrelated and coherent signals in low signal-to-noise ratio and limited snapshots. Making full use of the characteristics of moduli of eigenvalues, the DOAs of the uncorrelated signals are firstly estimated. Afterwards, their contributions are eliminated by means of spatial difference technique. Finally, in order to improve resolution and accuracy DOA estimation of remaining coherent signals while avoiding the cross-terms effect, a new beamforming solution based iterative adaptive approach (IAA) is proposed to deal with a reconstructed covariance matrix. The proposed method combines the advantages of both spatial difference method and the IAA algorithm while avoiding their shortcomings. Simulation results validate its effectiveness; meanwhile, the good performances of the proposed method in terms of resolution probability, detection probability, and estimation accuracy are demonstrated by comparison with the existing methods.
\end{abstract}

\section{Introduction}

Passive bistatic radar (PBR) exploiting illuminators of opportunity as uncooperative transmitters has become an emerging technology because it allows target detection and localization with advantages such as low cost, covert detection, and low vulnerability to electronic jamming $[1,2]$. However, these researches mainly focus on FM radio broadcasting [3, 4], mobile network base station [5], navigation satellites [6], digital video broadcasting, and digital audio broadcasting [7-9]. Compared with the aforementioned illuminators of opportunity, a dedicated radar usually has high power and an idea ambiguity function. In recent years, frequency agility and phased array technology are widely used in very high frequency (VHF) radar systems for remote sensing and surveillance purpose because it provides flexible beam scanning, improved detection probability, and high valuable counter to stealth technology $[10,11]$. The use of frequency agile phased array VHF radar can extend the range of available illuminators of opportunity, while it also poses many challenges in PBR signal processing, such as low signal-to-noise ratio (SNR), limited snapshots, etc. Thus, some complex signal processing algorithms must be employed, which increase the complexity of the systems. Due to multipath propagation, the direct signal of target and its multipath signal are coherent and closely spaced in the mainlobe. Meanwhile, the transmitting signals emitted from the exploited illuminator are received simultaneously, and they may come from the same direction as the direct signal. These problems significantly degrade the direction-of-arrival (DOA) estimation performance and even result in a failure of target detection and localization.

At present, many high resolution algorithms have been developed to cope with the scenario when uncorrelated and coherent signals coexist. The most popular solutions are the forward/backward spatial smoothing techniques [1215]. The problem, however, is that the decorrelation via spatial smoothing technique is acquired at the cost of the reduction in array aperture, which would further increase the width of mainlobe and make the resolution worse. Besides, these methods cannot resolve the uncorrelated and coherent signals that are in the same direction. Spatial difference 


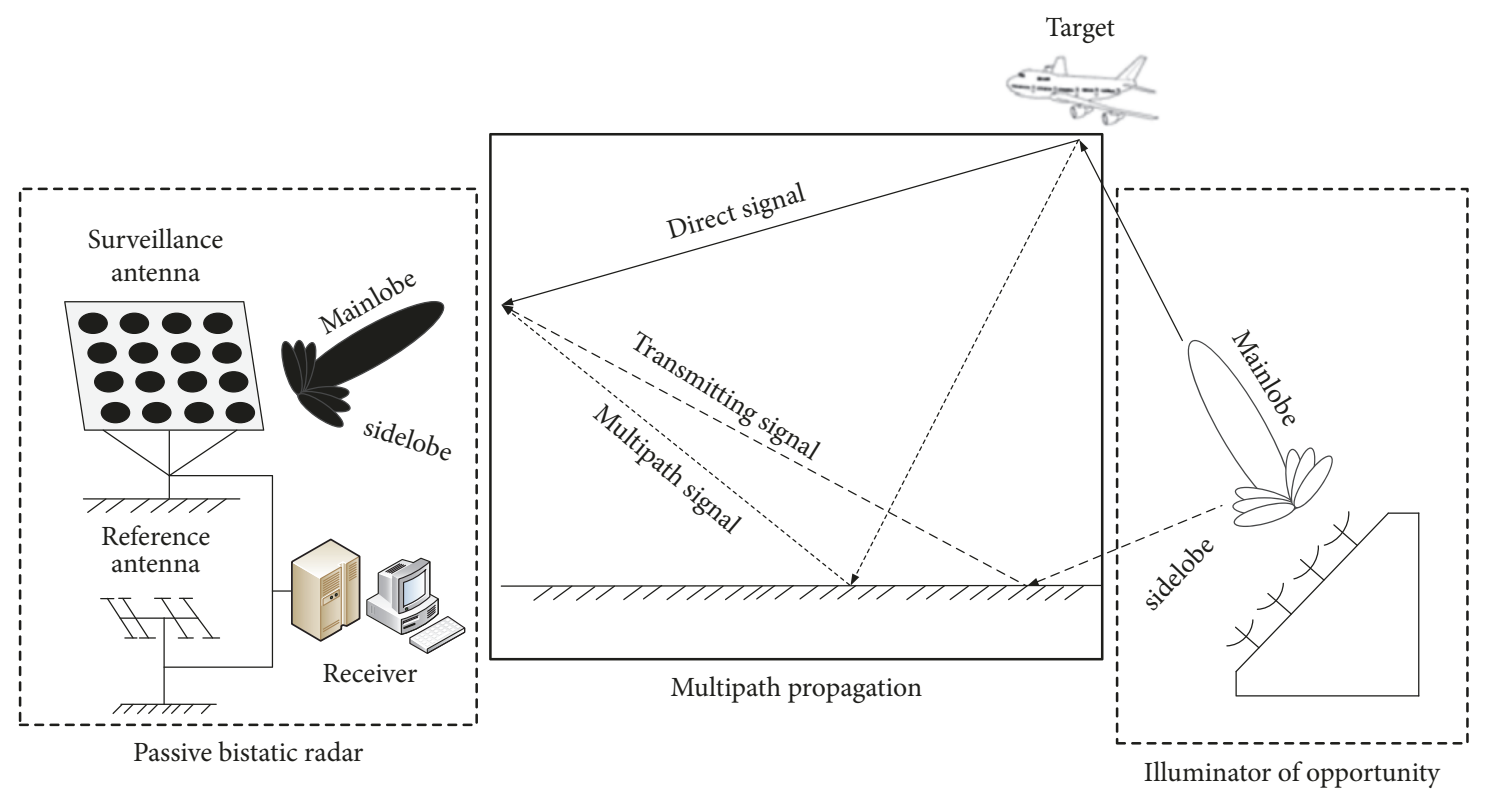

FIGURE 1: Basic operating scenario of PBR system.

methods are proposed to separately estimate the DOAs of the uncorrelated and coherent signals in the multipath environment [16-25]. These methods firstly estimate the DOAs of the uncorrelated signals and then eliminate their contribution using spatial difference technique. After that, the DOAs of the remaining coherent signals are estimated. However, the method in [16] is difficult in realization because the covariance matrix of the uncorrelated signals should be known as a priori. The method in [17] suffers from rank deficient problem. The required number of snapshots is too high to put $[18,19]$ into application. Since [20-23] eliminate the contributions of uncorrelated signals by constructing a difference matrix, which encounter the problem of crossterms effect and may yield wrong DOA estimates when the number of snapshots is small, the method introduced in [24, 25] makes full use of the property of the moduli of eigenvalues to resolve the uncorrelated signals from the coherent signals. However, the main disadvantage is that the DOA estimation performance degrades significantly in the low SNR and limited snapshots. Recently, an iterative adaptive approach (IAA) has attracted much attention [26-28]. A number of variant IAA-based beamforming solutions have shown a superresolution performance in dealing with closely spaced signals and work well with few numbers of snapshots [29]. Unfortunately, when uncorrelated and coherent signals are in the same direction, these methods fail to work, either. Combining the above analysis, we can get some conclusions that little literature has been published up to now in this field, partly because of the difficulties in PBR signal processing.

Motivated by previous work, we propose a new DOA estimation method in multipath environment for PBR. By making full use of the property of moduli of eigenvalues, the DOAs of the uncorrelated signals are estimated. Then the contribution of the uncorrelated signals is eliminated by using spatial difference technique; that is, only coherent signals remain in the spatial difference matrix. To eliminate the cross-terms effect while improving the resolution and accuracy of DOA estimation of the remaining coherent signals, a new IAA-based beamforming solution is conducted on a reconstructed covariance matrix. Simulation results validate the better performance of the proposed method by comparison with the existing algorithms.

\section{Problems Statement}

The basic operating scenario of PBR system is illustrated in Figure 1. As shown in the dashed line box, the PBR system consists of a surveillance antenna, a reference antenna, and a receiver. Meanwhile, a frequency agile phased array VHF radar is used as the uncooperative illuminator of opportunity. Refer to analysis in [10]; the frequency agility technology can increase the ability of antijamming and increase the detection probability. The phased array technology provides flexible beam pattern, angular diversity, and more degrees of freedom, which makes it competent for the task of detecting and tracking different targets.

However, the types of illuminator are enriched, while the difficulties for PBR signal processing also come along. In case of PBR exploiting uncooperative illuminator of opportunity as the transmitter, there is a problem that the instantaneous parameters of transmitting signal are unknown at the receiver; thus, a dedicated reference antenna needs to steer toward the uncooperative illuminator to receive the transmitting signal. Meanwhile, the frequency agility technology destroys the coherency between pulses and the ability of rapidly changing beam scanning makes it impossible for PBR to predict the next beam position. Therefore, these problems make the SNR become low and the number of snapshots is finite.

Furthermore, among the characteristics of the environment, the multipath propagation in VHF band is serious. As shown in the solid line box, the received signal is a 
mixture of transmitting signal of the exploited illuminator, the direct signal of target, and its multipath signal. The direct signal and multipath signal are coherent and closely spaced in the mainlobe. Meanwhile, the transmitting signal is received simultaneously and may come from the same direction as the direct signal.

Therefore, under such scenarios, the problems of DOA estimation in multipath environment for PBR system are summarized as how to deal with the closely spaced uncorrelated and coherent signals with high resolution and accuracy in the low SNR with limited snapshots.

\section{Signal Model}

Without loss of generality, in PBR scenario, a uniform linear array (ULA) with $N$ isotropic sensors is considered as the surveillance antenna. Let the first sensor be the reference, and take $\theta$ as the DOA of an impinging signal. Then, the steering vector can be expressed as

$$
\boldsymbol{a}(\theta)=\left[1, e^{-(\mathrm{j} 2 \pi d \sin \theta) / \lambda}, \cdots, e^{-(\mathrm{N}-1)((\mathrm{j} 2 \pi d \sin \theta) / \lambda)}\right]^{T}
$$

where $\lambda$ is the wavelength of the signal, $d$ is the distance between adjacent sensors, and the superscript $T$ denotes the transpose operator.

Assume that $K$ far-field narrowband signals are received simultaneously and are mixtures of $K_{U}$ uncorrelated signals and $K_{C}$ coherent signals with several groups. The $K_{U}$ signals are uncorrelated, and the signal that comes from direction $\theta_{k}$ corresponding to the signal $s_{k}$ with power $\sigma_{k}^{2}, k=1, \cdots, K_{U}$. The remaining $K_{C}$ coherent signals are divided into $C$ groups, which come from $C$ statistically independent signals with power $\sigma_{k}^{2}, k=K_{U}+1, \ldots, K_{U}+C$, and with $L_{k}$ multipath signals for each signal. That is, the relationship between uncorrelated and coherent signals is

$$
K=K_{U}+K_{C}, \quad K_{C}=\sum_{k=K_{U}+1}^{K_{U}+C} L_{k}
$$

In the $k t h$ coherent group, the multipath signal that comes from the direction $\theta_{k, l}, l=1, \cdots, L_{k}$ corresponds to the lth multipath propagation of the independent signal and its complex reflection coefficient is $\rho_{k, l}$. It is assumed that the uncorrelated signals and coherent signals in different groups are regarded as uncorrelated with each other. Consequently, the model of the received signals is given by

$$
\begin{aligned}
\boldsymbol{x}(t)= & \sum_{k=1}^{K_{U}} \boldsymbol{a}\left(\theta_{k}\right) s_{k}(t) \\
& +\sum_{k=K_{U}+1 \boldsymbol{l}=\mathbf{1}}^{K_{U+} C} \sum_{k}^{\boldsymbol{L}_{k}} \boldsymbol{a}\left(\theta_{k, l}\right) \rho_{k, l} s_{k}(t)+\boldsymbol{n}(t) \\
= & \boldsymbol{A}_{U} \boldsymbol{s}_{U}(t)+\boldsymbol{A}_{C} \boldsymbol{s}_{C}(t)+\boldsymbol{n}(t)=\boldsymbol{A s}(t)+\boldsymbol{n}(t) \\
= & \widehat{\boldsymbol{A}} \Upsilon \boldsymbol{s}(t)+\boldsymbol{n}(t)
\end{aligned}
$$

where $\boldsymbol{A}=\left[\boldsymbol{A}_{U}, \boldsymbol{A}_{C}\right], \boldsymbol{A}_{U}=\left[\boldsymbol{a}\left(\theta_{1}\right), \cdots, \boldsymbol{a}\left(\theta_{K_{U}}\right)\right]$, and $\boldsymbol{A}_{C}=$ $\left[\widetilde{\boldsymbol{A}}_{K_{U}+1} \boldsymbol{\rho}_{K_{U}+1}, \cdots, \widetilde{\boldsymbol{A}}_{K_{U}+1} \boldsymbol{\rho}_{K_{U}+1}\right]$ are the array flow pattern.
$\widetilde{\boldsymbol{A}}_{k}=\left[\boldsymbol{a}\left(\theta_{k, 1}\right), \cdots, \boldsymbol{a}\left(\theta_{k, L_{k}}\right)\right]$ and $\boldsymbol{\rho}_{k}=\left[\rho_{k, 1}, \cdots, \rho_{k, L_{k}}\right]^{T}$ contain the reflection coefficient of $k t h$ multipath propagation. $\widehat{\boldsymbol{A}}=\left[\boldsymbol{A}_{U}, \widetilde{\boldsymbol{A}}_{K_{U}+1}, \cdots, \widetilde{\boldsymbol{A}}_{K_{U}+C}\right] ; \Upsilon \mathbf{\Upsilon}=$ $\operatorname{blkdiag}\left\{\boldsymbol{I}_{K_{U}}, \boldsymbol{\rho}_{K_{U}+1}, \cdots, \boldsymbol{\rho}_{K_{U}+C}\right\} . \boldsymbol{s}(t)=\left[\boldsymbol{s}_{U}^{T}(t), \boldsymbol{s}_{C}^{T}(t)\right]^{T}$, $\boldsymbol{s}_{U}(t)$ is the source vector of the uncorrelated signals, and $\boldsymbol{s}_{\boldsymbol{C}}(t)$ is the source vector of the coherent groups. $\boldsymbol{n}(t)$ is the additive Gaussian white noise with mean zero and variance $\sigma_{n}^{2}$, which is uncorrelated with each other and the signals. Besides, the operator blkdiag represents a block diagonal matrix.

After that, the array covariance matrix can be obtained

$$
\boldsymbol{R}=E\left\{\boldsymbol{x}(t) \boldsymbol{x}^{\boldsymbol{H}}(t)\right\}=\boldsymbol{A}_{U} \boldsymbol{R}_{U} \boldsymbol{A}_{U}^{H}+\boldsymbol{A}_{C} \boldsymbol{R}_{C} \boldsymbol{A}_{C}^{H}+\boldsymbol{\sigma}_{n}^{2} \boldsymbol{I}_{N}
$$

where $\boldsymbol{R}_{U}=\operatorname{diag}\left\{\sigma_{1}^{2}, \cdots, \sigma_{K_{U}}^{2}\right\}$ is the covariance matrix of uncorrelated signals and $\boldsymbol{R}_{C}=\operatorname{diag}\left\{\sigma_{K_{U}+1}^{2}, \cdots, \sigma_{K_{U+C}}^{2}\right\}$ is the covariance matrix of coherent signals. $\boldsymbol{I}_{N}$ stands for the $N \times N$ identity matrix. Besides, the superscript $H$ denotes the transpose operator; the operator $\operatorname{diag}\left\{f_{1}, \cdots, f_{N}\right\}$ represents a diagonal matrix with the diagonal entry $f_{k}, k=1, \cdots, N$.

\section{The Proposed DOA Estimation Method}

4.1. DOA Estimation of Uncorrelated Signals. For the DOA estimation of the uncorrelated signals, the eigenvalue decomposition (EVD) of the covariance matrix $\boldsymbol{R}$ is conducted and the result is given

$$
\boldsymbol{R}=\boldsymbol{U}_{S} \boldsymbol{\Lambda}_{S} \boldsymbol{U}_{S}^{H}+\boldsymbol{U}_{N} \boldsymbol{\Lambda}_{N} \boldsymbol{U}_{N}^{H}
$$

where $\boldsymbol{U}_{S}=\left[\boldsymbol{u}_{1}, \cdots, \boldsymbol{u}_{K_{U}+C}\right], \boldsymbol{\Lambda}_{S}=\operatorname{diag}\left\{\lambda_{1}, \cdots, \lambda_{K_{U}+C}\right\}$, $\boldsymbol{U}_{N}=\left[\boldsymbol{u}_{K_{U}+C+1}, \cdots, \boldsymbol{u}_{N}\right]$, and $\boldsymbol{\Lambda}_{N}=\operatorname{diag}\left\{\lambda_{K_{U}+C+1}, \cdots, \lambda_{N}\right\}$. The $U_{S}$ is signal subspace matrix whose columns are the eigenvectors corresponds to $K_{U}+C$ largest eigenvalues; $\boldsymbol{U}_{N}$ is noise subspace composed of the eigenvectors correspond to $N-\left(K_{U}+C\right)$ smallest eigenvalues. $\Lambda_{S}$ and $\boldsymbol{\Lambda}_{N}$ are the diagonal matrixes constituted by their eigenvalues.

Moreover, the $\boldsymbol{U}_{S}$ is also spanned by the $\widehat{A} \Upsilon[24,25]$; thus it is written as

$$
U_{S}=\widehat{A} \Upsilon \mathbf{T}
$$

where $\boldsymbol{T}$ is a full-rank matrix. The $\boldsymbol{U}_{S}$ can be partitioned into two overlapped submatrixes with the size of $(N-1) \times\left(K_{U}+C\right)$, which are expressed as

$$
\begin{aligned}
& \boldsymbol{U}_{\mathbf{1}}=\boldsymbol{U}_{S}(1: N-1,:)=\widehat{A}_{\mathbf{1}} \mathbf{Y T} \\
& \boldsymbol{U}_{\mathbf{2}}=\boldsymbol{U}_{S}(2: N,:)=\widehat{A}_{\mathbf{2}} \boldsymbol{Y T}=\widehat{A}_{\mathbf{1}} \boldsymbol{\Phi} \mathbf{Y T}
\end{aligned}
$$

where $\widehat{\boldsymbol{A}}_{1}$ and $\widehat{\boldsymbol{A}}_{2}$ are the first and last $N-1$ rows of $\widehat{\boldsymbol{A}}$. $\Phi=\operatorname{diag}\left\{\phi_{1}, \cdots, \phi_{K}\right\}$ with $\phi_{k}=e^{-\left(j 2 \pi d \sin \theta_{k}\right) / \lambda}, k=1, \cdots, K$. Afterwards, a new matrix is constructed as

$$
\boldsymbol{U}=\boldsymbol{U}_{\mathbf{1}}^{\dagger} \boldsymbol{U}_{\mathbf{2}}=\mathbf{T}^{-1} \boldsymbol{Y}^{\dagger} \boldsymbol{\Phi} \mathbf{Y} \mathbf{T}=\mathbf{T}^{-1} \boldsymbol{\Lambda}_{T} \mathbf{T}
$$

where the superscript $\uparrow$ denotes the matrix pseudo inverse operator and the matrixes and vectors in (9) have the following forms:

$$
\begin{aligned}
& \boldsymbol{Y}^{\dagger}=\operatorname{blkdiag}\left\{\boldsymbol{I}_{K_{U}}, \boldsymbol{\eta}_{K_{U}+\mathbf{1}}, \cdots, \boldsymbol{\eta}_{K_{U}+C}\right\}, \\
& \boldsymbol{\eta}_{k}=\left[\eta_{k, 1}, \cdots, \eta_{k, L_{k}}\right]
\end{aligned}
$$


and

$$
\begin{aligned}
\boldsymbol{\Lambda}_{T} & =\operatorname{diag}\left\{v_{1}, \cdots, v_{K_{U}}, v_{K_{U}+1}, \cdots, v_{K_{U}+C}\right\} \\
v_{k} & = \begin{cases}e^{-\left(j 2 \pi d \sin \theta_{k}\right) / \lambda}, & k=1, \cdots, K_{U} \\
\eta_{k, 1} e^{-\left(j 2 \pi d \sin \theta_{k, 1}\right) / \lambda}+\eta_{k, 1} \rho_{k, 1} e^{-\left(j 2 \pi d \sin \theta_{k, 2}\right) / \lambda}+\cdots+\eta_{k, L_{k}} \rho_{k, L_{k-1}} e^{-\left(j 2 \pi d \sin \theta_{k, L_{k}}\right) / \lambda}, & k=K_{U}+1, \cdots, K_{U}+C\end{cases}
\end{aligned}
$$

Based on the property of the diagonal elements, which is well documented in [24], and we can obtain the following results:

$$
\begin{aligned}
\left|v_{K_{U}+C}\right| & \leq\left|v_{K_{U}+C-1}\right| \leq \cdots \leq\left|v_{K_{U}+1}\right|<\left|v_{K_{U}}\right|=\cdots \\
& =\left|v_{2}\right|=\left|v_{1}\right|=1
\end{aligned}
$$

Clearly, by performing EVD of the $\boldsymbol{U}$, the moduli values of the eigenvalues corresponding to the uncorrelated signals are equal to 1 , and the moduli values of the eigenvalues corresponding to the coherent signals are all less than 1 . Therefore, we can choose a threshold $\xi$ to resolve the uncorrelated signals from the coherent signals. In the meanwhile, the number of uncorrelated signals is estimated by sorting the $\left|v_{k}\right|, k=1, \cdots, K_{U}+C$ in descending order and substituting them into the following equation:

$$
\delta_{k}=|| v_{k}|-1|
$$

Thus, if the $\left|v_{k}\right|$ is the first to satisfy $\delta_{k}>\xi$, the number of the uncorrelated signals is $k-1$, and the corresponding DOA is obtained as follows:

$$
\theta_{k}=\sin ^{-1}\left(-\frac{\lambda}{j 2 \pi d} \operatorname{angle}\left(v_{k}\right)\right)
$$

4.2. DOA Estimation of Coherent Signals. The DOAs of the remaining signals are then estimated by using a novel IAAbased beamforming solution. Herein, the equation in (4) is rewritten as

$$
\boldsymbol{R}=E\left\{\boldsymbol{x}(t) \boldsymbol{x}^{H}(t)\right\}=\overline{\boldsymbol{R}}_{U}+\overline{\boldsymbol{R}}_{C}+\boldsymbol{\sigma}_{n}^{2} \boldsymbol{I}_{N}
$$

in $(16)$, the $(i, j)$ element in the covariance matrix of uncorrelated signals can be expressed as

$$
\overline{\boldsymbol{R}}_{U}(i, j)=\sum_{k=1}^{K_{U}} \sigma_{k}^{2} \phi_{k}^{i-j}
$$

Apparently, $\overline{\boldsymbol{R}}_{U}$ is a Toeplitz matrix, according to the property that any Toeplitz matrix $\boldsymbol{\Xi}$ will satisfy the fact that

$$
J \Xi=\Xi
$$

where $\boldsymbol{J}$ is the exchange matrix, which has unity elements along the counter-diagonal and zeros elsewhere. Thus, the uncorrelated signals can be eliminated by means of spatial difference technique [17], which can be expressed as

$$
\begin{aligned}
\boldsymbol{R}_{d} & =\boldsymbol{R}-\boldsymbol{J} \boldsymbol{R}^{T} \boldsymbol{J} \\
& =\left(\overline{\boldsymbol{R}}_{U}+\overline{\boldsymbol{R}}_{C}+\boldsymbol{\sigma}_{n}^{2} \boldsymbol{I}_{N}\right)-\boldsymbol{J}\left(\overline{\boldsymbol{R}}_{U}+\overline{\boldsymbol{R}}_{C}+\boldsymbol{\sigma}_{n}^{2} \boldsymbol{I}_{N}\right)^{T} \boldsymbol{J} \\
& =\overline{\boldsymbol{R}}_{C}-\boldsymbol{J} \overline{\boldsymbol{R}}_{C}^{T} \boldsymbol{J}
\end{aligned}
$$

Theoretically, only coherent signals remain in the spatial differencing matrix in this step. However, in the practical PBR application scenario, the noise cannot be eliminated completely. Furthermore, the EVD of $\boldsymbol{R}_{d}$ would generate cross-terms effect, which would result in a high probability of failure in DOA estimation of closely spaced coherent signals.

To solve the aforementioned problems, we propose a new IAA-based beamforming solution with respect to an improved covariance matrix. Then, we rewrite (4) as

$$
\boldsymbol{R}=E\left\{\boldsymbol{x}(t) \boldsymbol{x}^{H}(t)\right\}=\overline{\boldsymbol{A}} \overline{\boldsymbol{P}} \overline{\boldsymbol{A}}
$$

where $\overline{\boldsymbol{A}}$ and $\overline{\boldsymbol{P}}$ denote the reconstructed array flow pattern and signal power matrix, which can be expressed as

$$
\begin{aligned}
\overline{\boldsymbol{A}} & =\left[\boldsymbol{A}_{U}, \boldsymbol{A}_{C}, \boldsymbol{I}_{\boldsymbol{N}}\right]=\left[\boldsymbol{a}\left(\theta_{1}\right), \cdots, \boldsymbol{a}\left(\theta_{K_{U}}\right), \boldsymbol{a}\left(\theta_{K_{U}+1}\right)\right. \\
& \left.\cdot \boldsymbol{\rho}_{K_{U}+1}, \cdots, \boldsymbol{a}\left(\theta_{K_{U}+C}\right) \boldsymbol{\rho}_{K_{U}+C}, \boldsymbol{I}_{\boldsymbol{N}}\right] \\
& =\left[\overline{\boldsymbol{a}}\left(\theta_{1}\right), \cdots, \overline{\boldsymbol{a}}\left(\theta_{K}\right), \cdots, \overline{\boldsymbol{a}}\left(\theta_{K+N}\right)\right]
\end{aligned}
$$

and

$$
\begin{aligned}
\overline{\boldsymbol{P}} & =\operatorname{blkdiag}\left\{\boldsymbol{P}_{U}, \boldsymbol{P}_{C}, \boldsymbol{\sigma}_{n}^{2} \boldsymbol{I}_{N}\right\} \\
& =\operatorname{diag}\left\{\bar{P}_{1}, \cdots, \bar{P}_{K}, \cdots, \bar{P}_{K+N}\right\}
\end{aligned}
$$

where the diagonal value $\bar{P}_{k}$ represents the corresponding signal power at each angle.

Afterwards, according the IAA algorithm [27] (see Appendix), the IAA-based beamformer for each DOA of the remaining coherent signals is computed as

$$
\boldsymbol{w}_{k}=\frac{\boldsymbol{R}^{-1} \overline{\boldsymbol{a}}\left(\theta_{k}\right)}{\overline{\boldsymbol{a}}^{H}\left(\theta_{k}\right) \boldsymbol{R}^{-1} \overline{\boldsymbol{a}}\left(\theta_{k}\right)}
$$

Therefore, the estimated signal power of corresponding direction can be obtained

$$
\widehat{P}_{k}=\boldsymbol{w}_{k}^{H} \boldsymbol{R}_{d} \boldsymbol{w}_{k}
$$


In order to eliminate the cross-terms effect while improving the resolution and accuracy of DOA estimation, a new covariance matrix is constructed by squaring the spatial difference matrix [21]

$$
\boldsymbol{R}_{D}=\boldsymbol{R}_{d} \boldsymbol{R}_{d}^{H}
$$

Then, the spatial spectrum of each direction is obtained by replacing $\boldsymbol{R}_{d}$ with $\boldsymbol{R}_{D}$

$$
\widetilde{P}_{k}=\boldsymbol{w}_{k}^{H} \boldsymbol{R}_{D} \boldsymbol{w}_{k}
$$

As a result, by performing the IAA algorithm with respect to the reconstructed covariance matrix, the DOAs of the remaining coherent signals are estimated.

4.3. Summary and Discussion of the Proposed Method. In this subsection, the proposed method is summarized and discussed as follows:

(i) Since the pervious analysis of practical PBR application scenario, the covariance matrix of the received signal is unavailable: thus it is replaced by the sample-average estimated array autocovariance matrix.

$$
\widehat{\boldsymbol{R}}=\frac{1}{N_{P}} \sum_{n=1}^{N_{P}} \boldsymbol{x}(\mathrm{n}) \boldsymbol{x}^{H}(n)
$$

where $\boldsymbol{x}(n)$ is the received signal of the $n t h$ snapshot: $N_{P}$ is the number of snapshot.

(ii) Then, by performing the EVD of the matrix $\widehat{\boldsymbol{R}}$, the signal subspace $U_{S}$ is obtained, and the $U_{1}$ and $U_{2}$ are computed to construct $\boldsymbol{U}$. Thus, the moduli of eigenvalues of $U$ are obtained.

(iii) Based on the property of moduli of eigenvalues, the uncorrelated signals are resolved from coherent signals while the DOAs of the uncorrelated signals are estimated

(iv) To eliminate the contribution of uncorrelated signals and improve the resolution and accuracy DOA estimation of coherent signals, the spatial difference matrix $\boldsymbol{R}_{d}$ is computed and a new covariance matrix $\boldsymbol{R}_{D}$ is constructed by squaring the spatial difference matrix.

(v) Finally, the DOAs of remaining coherent signals are estimated by performing IAA algorithm on the reconstructed covariance matrix $\boldsymbol{R}_{D}$.

It is noteworthy that our proposed method combines the advantages of both spatial difference method and the IAA algorithm while avoiding their disadvantages. When the uncorrelated and coherent signals coexist in multipath environment, especially the uncorrelated signal coming from the same direction as the coherent signals do, the method can still resolve them, whereas the IAA algorithm fails to work. The performance of the spatial difference method degrades significantly in the low SNR with limited snapshots, but our proposed method can still work well. Besides, by exploiting our method, the DOAs of uncorrelated and coherent signals are estimated separately, which can be parallelized easily to speed up the processing in practical application.

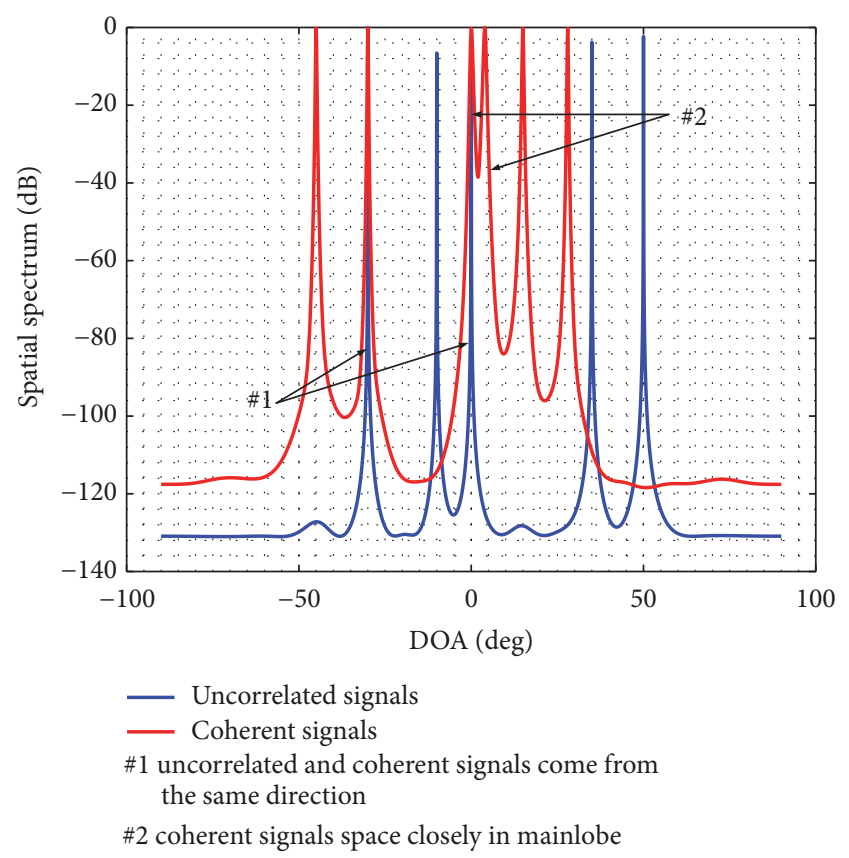

FIGURE 2: Spatial spectrums of the uncorrelated and coherent signals.

\section{Simulation Results}

In this section, the performance of the proposed method is investigated by simulation experiments. For PBR system, a ULA of $N=16$ omnidirectional antenna sensors spaced halfwavelength interspacing is used as the surveillance antenna and the half power width of mainlobe can be computed as $\theta_{0.5} \approx 6.4^{\circ}$. In the simulations, the threshold is set as $\xi=0.05$ to resolve uncorrelated signals from coherent signals, and the scanning size is uniform in the range from $-90^{\circ}$ to $90^{\circ}$ with $0.1^{\circ}$ increment.

In the first simulation, we consider five uncorrelated signals from $\left[-30^{\circ},-10^{\circ}, 0^{\circ}, 35^{\circ}, 50^{\circ}\right]$ and two groups of coherent signals from $\left[0^{\circ}, 4^{\circ}\right]$ and $\left[-45^{\circ},-30^{\circ}, 15^{\circ}, 28^{\circ}\right]$. The reflection coefficient of the first group of coherent signals is $[1,0.3837+0.9017 j]$ and the other group is $[1,-0.7513+$ $0.0579 j,-0.3427-0.3155 j, 0.5873-0.7385 j]$. For simplicity, all signals are of equal power. It is worth pointing out that the two uncorrelated signals are set as $\theta=-30^{\circ}$ and $=0^{\circ}$, which come from the same direction with the two groups of coherent signals; in the first group of coherent signals, the two signals are closely spaced in the mainlobe. The simulation is carried out in the SNR of $10 \mathrm{~dB}$ and the number of snapshots of 100 . Therefore, the spatial spectrum of the uncorrelated and coherent signals is shown in Figure 2. As is apparent, the proposed method can easily resolve the uncorrelated signals from the coherent signal in same direction, and it also has satisfactory performance in resolving closely spaced coherent signals.

The second simulation studies the superresolution performance of two closely spaced signals with the proposed method. In the simulation, we define the probability of resolution as

$$
P_{r}=\frac{\Omega_{r}}{\Omega}
$$




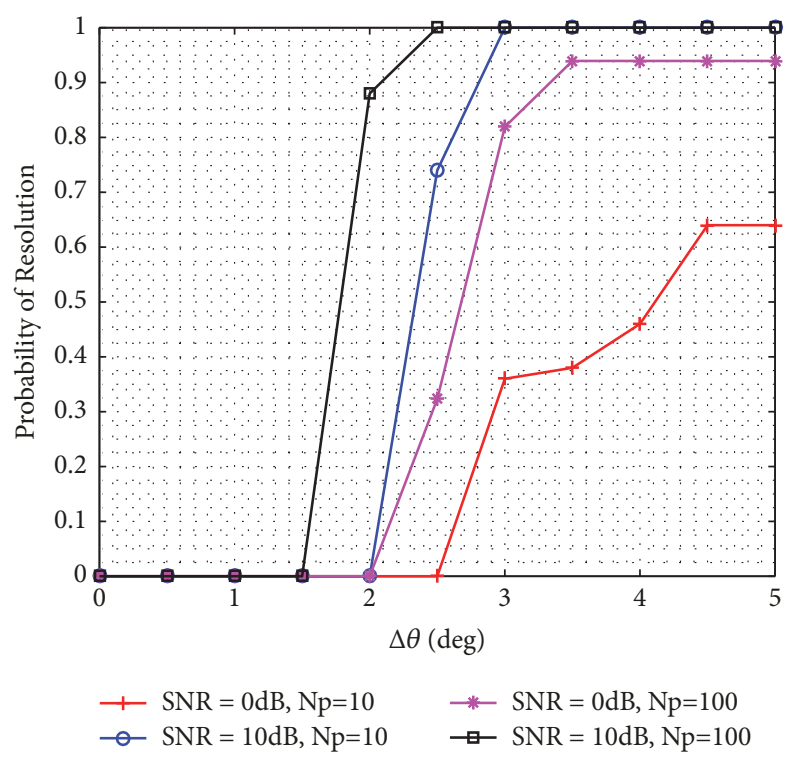

FIgURE 3: The resolution probability versus the angular gap.

where $\Omega_{r}$ and $\Omega$ represent the times of successful resolution result and the total trials, respectively.

The angular gap between two closely spaced coherent signals is defined as $\theta=\left|\theta_{2}-\theta_{1}\right|$, and when the condition of $\left(P_{r}\left(\theta_{2}\right)+P_{r}\left(\theta_{1}\right)\right) / 2>P_{r}\left(\left(\theta_{1}+\theta_{2}\right) / 2\right)$ is satisfied, the result is considered successful. Herein, we set the DOA of the first signal as $0^{\circ}$, and the other one vary from $0^{\circ}$ to $5^{\circ}$. Under such simulation scenario, the coherent signals are always in the mainlobe. Figure 3 shows the curves of probability of resolution with respect to the SNR of $0 \mathrm{~dB}$ and $10 \mathrm{~dB}$, the number of snapshots of 10 and 100. It is shown that our proposed method has a little performance degradation in the low SNR, but the overall performance is satisfactory as the SNR is larger. Especially, when the angular gap is $3^{\circ}$, the probability of resolution of is almost equal to 1 in $\mathrm{SNR}=10 \mathrm{~dB}$ and $N_{P}=10$. Therefore, the superresolution DOA estimation performance of the proposed method is verified.

In the third simulation, we investigate the high accuracy performance of DOA estimation with our proposed method. The detection probability in the simulation is defined as

$$
P_{d}=\frac{\Omega_{d}}{\Omega}
$$

where $\Omega_{d}$ and $\Omega$ stand for the times of successful detection result of $K$ signals and the total tests, respectively.

The DOAs detection result is considered successful when the difference between the estimated DOA and the true DOA is less than $2^{\circ}$, i.e., $P_{d}=1$ subject to $\Delta \theta=\mid \widehat{\theta}_{k}-$ $\theta_{k} \mid \leq 2^{\circ}, k=1, \cdots, K$. The simulation considers the same scenario as the first one. The DOA estimation results with respect to the changes of SNR and the number of snapshots are plotted in Figures 4 and 5. Apparently, the curves of the detection probability indicate that our proposed method has good performance of DOA estimation when the SNR varies from $0 \mathrm{~dB}$ and $10 \mathrm{~dB}$, and the number of snapshots is higher than 30 . This is because that our proposed method

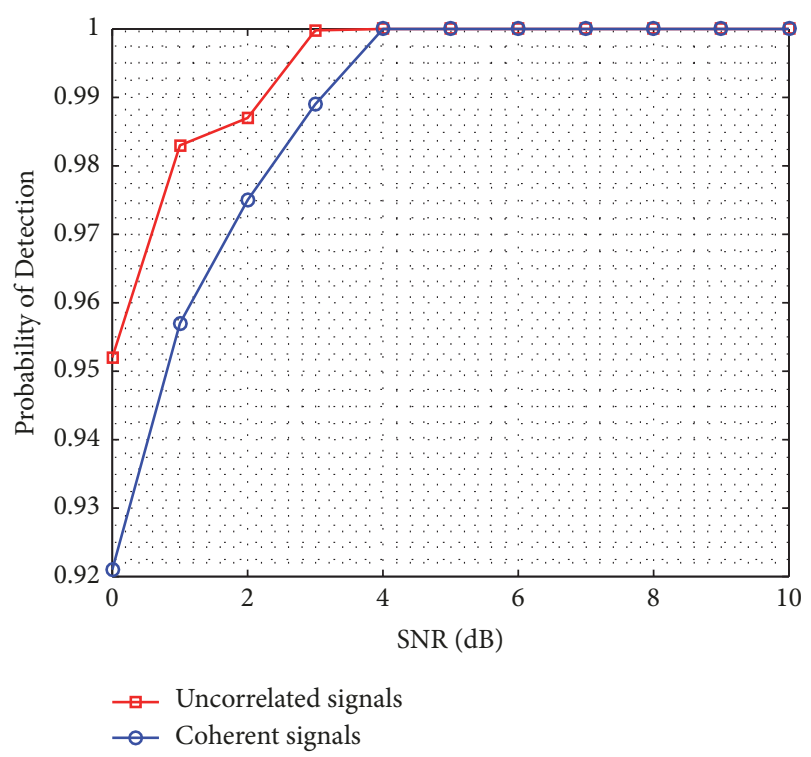

FIGURE 4: The detection probability versus the SNR with $N_{P}=100$.

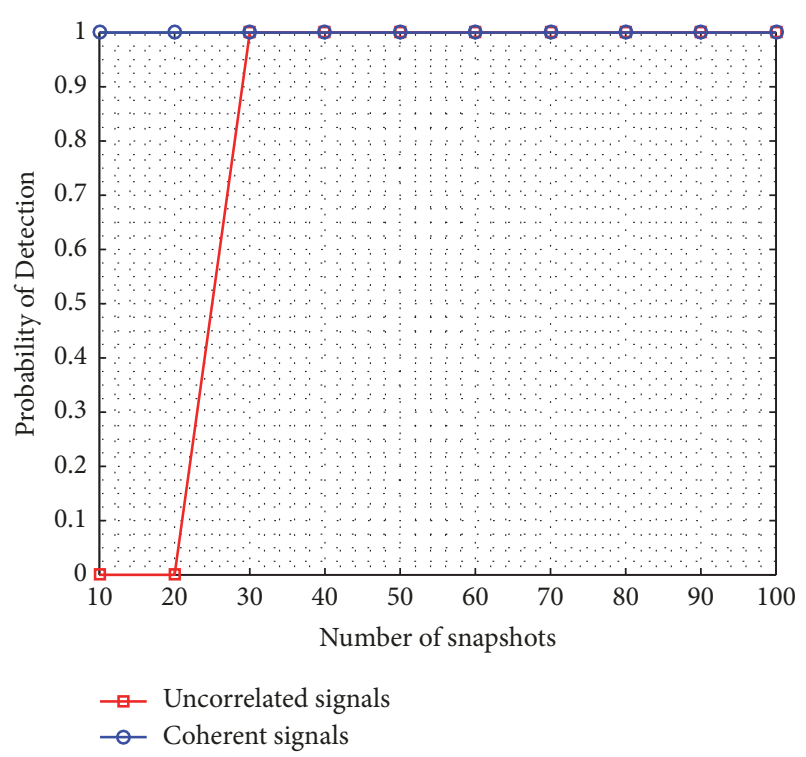

FIGURE 5: The detection probability versus the number of snapshots with SNR = 10dB.

makes full use of the true signal power when dealing with uncorrelated and coherent signals, which is different from the existing subspace-based high resolution algorithms. These methods use the orthogonality of the signal subspace and the noise subspace in a certain direction to calculate the spatial spectrum, and the performance of DOA estimation is poor when the SNR is low and the number of snapshots is small. Therefore, similar to the previous results, the proposed method has high accuracy DOA estimation performance in dealing with uncorrelated and coherent signals.

In the last simulation, we further examine the performance of the proposed method by comparison. We select the method in [23] and the method in [24] as the comparative 


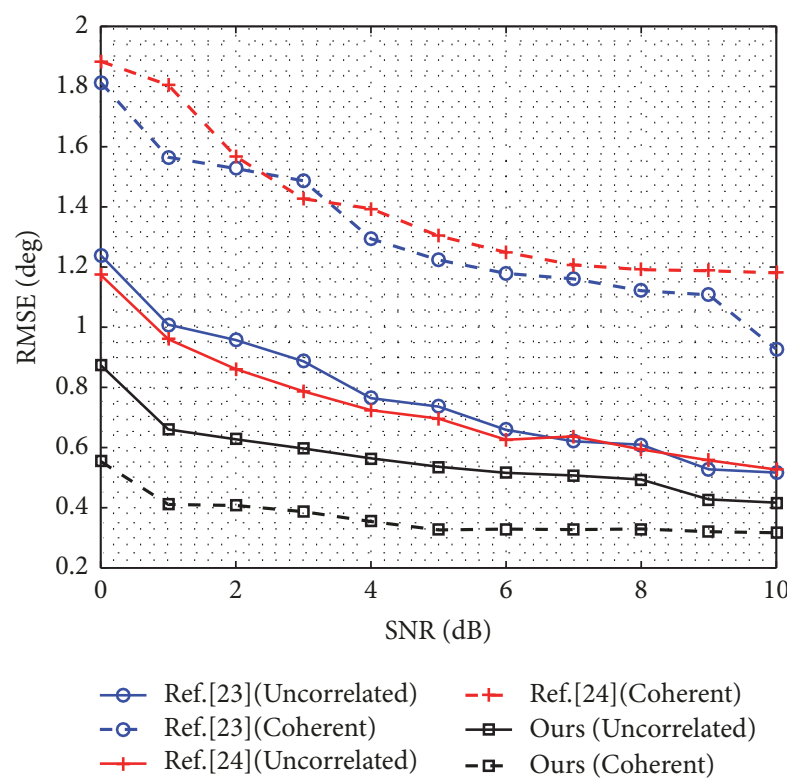

FIGURE 6: The RMSE of DOA estimation versus SNR.

methods. The performance is tested by the root mean square error (RMSE), which is defined as

$$
\operatorname{RMSE}=\sqrt{\frac{1}{200} \frac{1}{K} \sum_{\tau=1}^{200} \sum_{k=1}^{K}\left(\widehat{\theta}_{k}^{(\tau)}-\theta_{k}\right)^{2}}
$$

In each simulation scenario, 200 Monte Carlo runs are performed. Figures 6 and 7 show the RMSE curves of DOA estimation with respect to the SNR and the number of snapshots.

It can be seen from the RMSE curves of DOA estimation with different methods that the DOA estimation performance of our method is better than the method in [23] and the method in [24], especially in the low SNR with small number of snapshots. For method in [23], the reason of performance degradation is that it utilizes the whole array to estimate the uncorrelated signals and then uses the reduced array to estimate the coherent signals in a subsequent stage. When the coherent signals are decorrelated by using forward/backward spatial smoothing technique, the reduction in array aperture would broaden the mainlobe, which significantly degrades the resolution and accuracy of DOA estimation. For method in [24], the performance of DOA estimation of uncorrelated signals is similar to our method due to the same process in dealing with uncorrelated signals. However, when dealing with the remaining coherent signals, it also uses forward/backward spatial smoothing technique, and the performance is not satisfactory, either. For our method, when dealing with uncorrelated and coherent signals, we combine the advantages of spatial difference method and IAA algorithm and propose a novel IAA-based beamforming solution with respect to the improved covariance matrix, which can effectively improve the performance of resolution and accuracy in DOA estimation.

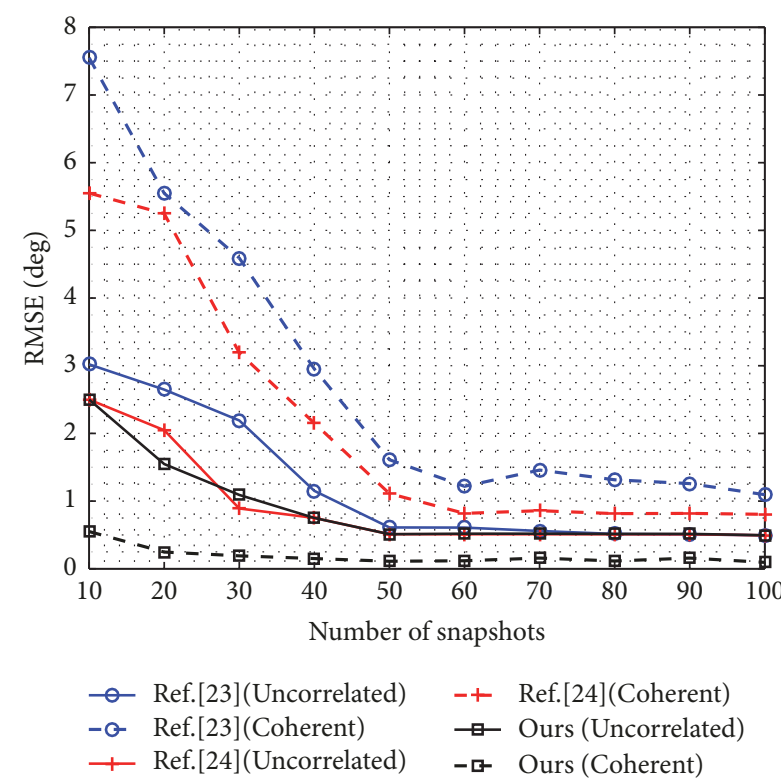

FIgURE 7: The RMSE of DOA estimation versus the number of snapshots.

\section{Conclusions}

In this paper, we propose a novel DOA estimation method in the multipath environment for PBR using frequency agile phased array VHF radar as illuminator of opportunity. The proposed method combines the advantages of spatial difference method and the IAA algorithm while avoiding their shortcomings and then performs a new IAA-based beamforming solution on an improved covariance matrix to deal with closely spaced uncorrelated and coherent signals. The simulation results show that it has satisfactory DOA estimation performance in the scenario of low SNR and small number of snapshots. Furthermore, in the proposed method, the DOAs of uncorrelated and coherent signals are estimated separately, which can be parallelized easily to speed up processing, and can be a viable solution for PBR applications. Since the presented PBR system operates in the VHF band, multipath propagation in complex terrain is serious and unavoidable. In order to further improve the performance of target detection and localization, the dynamic characteristics of electromagnetic and geographical environment and antimultipath technology should be taken into consideration in future works.

\section{Appendix}

The IAA algorithm is a data-dependent, nonparametric method which is based on a weighted least squares (WLS) approach. Firstly, we define the covariance matrix of noise based on the above sparse signal model

$$
\overline{\mathrm{Q}}_{k}=\boldsymbol{R}-\bar{P}_{k} \overline{\boldsymbol{a}}\left(\theta_{k}\right) \overline{\boldsymbol{a}}^{H}\left(\theta_{k}\right)
$$

Then, the WLS cost function is given by

$$
\mathscr{M}_{V}=\sum_{n=1}^{N}\left\|\boldsymbol{x}(n)-\boldsymbol{s}_{\boldsymbol{k}}(n) \overline{\boldsymbol{a}}\left(\theta_{k}\right)\right\|_{V}^{2}
$$


where $\|\boldsymbol{y}\|_{V}^{2}=\boldsymbol{y}^{H} \boldsymbol{V} \boldsymbol{y}$. In (A.2), we perform partial derivative operation of $\mathscr{M}_{V}$ with respect to $\boldsymbol{s}_{\boldsymbol{k}}(n)$ and make the result be zero to obtain that

$$
\widehat{\boldsymbol{s}}_{\boldsymbol{k}}(n)=\frac{\overline{\boldsymbol{a}}^{H}\left(\theta_{k}\right) \boldsymbol{V} \boldsymbol{x}(n)}{\overline{\boldsymbol{a}}^{H}\left(\theta_{k}\right) \boldsymbol{V} \overline{\boldsymbol{a}}\left(\theta_{k}\right)}
$$

Then we can compute the mean square error (MSE) as

$$
\begin{aligned}
\mathrm{MSE} & =E\left\{\left[\boldsymbol{s}_{\boldsymbol{k}}(n)-\widehat{\boldsymbol{s}}_{\boldsymbol{k}}(n)\right]\left[\boldsymbol{s}_{\boldsymbol{k}}(n)-\widehat{\boldsymbol{s}}_{\boldsymbol{k}}(n]^{H}\right\}\right. \\
= & {\left[\overline{\boldsymbol{a}}^{H}\left(\theta_{k}\right) \boldsymbol{V} \overline{\boldsymbol{a}}\left(\theta_{k}\right)\right]^{-1} \overline{\boldsymbol{a}}^{H}\left(\theta_{k}\right) \boldsymbol{V E}\left[\boldsymbol{n}(n) \boldsymbol{n}^{H}(n)\right] } \\
& \cdot \boldsymbol{V}^{H} \overline{\boldsymbol{a}}\left(\theta_{k}\right)\left[\overline{\boldsymbol{a}}^{H}\left(\theta_{k}\right) \boldsymbol{V} \overline{\boldsymbol{a}}\left(\theta_{k}\right)\right]^{-1} \\
& =\left[\overline{\boldsymbol{a}}^{H}\left(\theta_{k}\right) \boldsymbol{V} \overline{\boldsymbol{a}}\left(\theta_{k}\right)\right]^{-1} \overline{\boldsymbol{a}}^{H}\left(\theta_{k}\right) \boldsymbol{V} \overline{\mathrm{Q}}_{k} \boldsymbol{V}^{H} \overline{\boldsymbol{a}}\left(\theta_{k}\right) \\
& \cdot\left[\overline{\boldsymbol{a}}^{H}\left(\theta_{k}\right) \boldsymbol{V} \boldsymbol{a}\left(\theta_{k}\right)\right]^{-1}
\end{aligned}
$$

Suppose $Z_{1}, Z_{2}$ are a column vector and a row vector, and the inverse matrix of $Z_{2} Z_{2}^{H}$ exists. Then, we can get the following matrix inequality:

$$
\boldsymbol{Z}_{\mathbf{1}}^{H} \boldsymbol{Z}_{\mathbf{1}} \geq\left(\boldsymbol{Z}_{\mathbf{2}} \boldsymbol{Z}_{\mathbf{1}}\right)^{H}\left(\boldsymbol{Z}_{\mathbf{2}} \boldsymbol{Z}_{\mathbf{2}}^{H}\right)^{-1}\left(\boldsymbol{Z}_{\mathbf{2}} \boldsymbol{Z}_{\mathbf{1}}\right)
$$

Let $Z_{\mathbf{2}}=\overline{\boldsymbol{a}}^{H}\left(\theta_{k}\right) \overline{\mathbf{Q}}_{\boldsymbol{k}}^{-\mathbf{1} / \mathbf{2}} ; \boldsymbol{Z}_{\mathbf{1}}=\overline{\mathbf{Q}}_{\boldsymbol{k}}^{\mathbf{1} / \mathbf{2}} \boldsymbol{Z}_{\mathbf{3}}^{\boldsymbol{H}}$ with $\boldsymbol{Z}_{\mathbf{3}}=$ $\left[\overline{\boldsymbol{a}}^{H}\left(\theta_{k}\right) \boldsymbol{V} \overline{\boldsymbol{a}}\left(\theta_{k}\right)\right]^{-1} \overline{\boldsymbol{a}}^{H}\left(\theta_{k}\right) \boldsymbol{V}$, then we can rewrite (A.4) as

$$
\begin{aligned}
\text { MSE } & =\boldsymbol{Z}_{\mathbf{1}}^{H} \boldsymbol{Z}_{\mathbf{1}} \geq\left(\boldsymbol{Z}_{\mathbf{2}} \boldsymbol{Z}_{\mathbf{1}}\right)^{H}\left(\boldsymbol{Z}_{\mathbf{2}} \boldsymbol{Z}_{\mathbf{2}}^{\boldsymbol{H}}\right)^{-1}\left(\boldsymbol{Z}_{\mathbf{2}} \boldsymbol{Z}_{\mathbf{1}}\right) \\
& =\left[\overline{\boldsymbol{a}}^{H}\left(\theta_{k}\right) \overline{\mathbf{Q}}_{k}^{-\mathbf{1}} \overline{\boldsymbol{a}}\left(\theta_{k}\right)\right]^{-1}
\end{aligned}
$$

Clearly, we can get the conclusion that when $\boldsymbol{V}=\overline{\mathbf{Q}}_{k}^{-\mathbf{1}}$, the result of MSE is minimal.

After that, using the matrix inversion lemma to yield

$$
\begin{aligned}
\widehat{\boldsymbol{s}}_{\boldsymbol{k}}(n) & =\frac{\overline{\boldsymbol{a}}^{H}\left(\theta_{k}\right) \overline{\mathbf{Q}}_{\boldsymbol{k}}^{\mathbf{1}}}{\overline{\boldsymbol{a}}^{H}\left(\theta_{k}\right) \overline{\mathbf{Q}}_{\boldsymbol{k}}^{-\mathbf{1}} \overline{\boldsymbol{a}}\left(\theta_{k}\right)} \boldsymbol{x}(n) \\
& =\frac{\overline{\boldsymbol{a}}^{H}\left(\theta_{k}\right) \boldsymbol{R}^{-\mathbf{1}}}{\overline{\boldsymbol{a}}^{H}\left(\theta_{k}\right) \boldsymbol{R}^{-\mathbf{1}} \overline{\boldsymbol{a}}\left(\theta_{k}\right)} \boldsymbol{x}(n)=\boldsymbol{w}_{\boldsymbol{k}}^{\boldsymbol{H}} \boldsymbol{x}(n)
\end{aligned}
$$

Therefore, the beamformer for each direction of the corresponding signal is

$$
\boldsymbol{w}_{k}=\frac{\boldsymbol{R}^{-\mathbf{1}} \overline{\boldsymbol{a}}\left(\theta_{k}\right)}{\overline{\boldsymbol{a}}^{H}\left(\theta_{k}\right) \boldsymbol{R}^{-\mathbf{1}} \overline{\boldsymbol{a}}\left(\theta_{k}\right)}
$$

Since the $\boldsymbol{R}$ in IAA algorithm depends on the unknown signal power, it must be done in an iterative way. The initialization is implemented by conventional beamforming. It is worth noting that the matrix $\overline{\boldsymbol{P}}$ and $\boldsymbol{R}$ are updated from the previous iteration, not the data snapshot.

\section{Data Availability}

No data were used to support this study.

\section{Conflicts of Interest}

The authors declare that they have no conflicts of interest.

\section{Acknowledgments}

This work was partly supported by the National Natural Science Foundation of China under Grant 61401489.

\section{References}

[1] F. Colone, C. Bongioanni, and P. Lombardo, "Multifrequency integration in FM radio-based passive bistatic radar. Part I: target detection," IEEE Aerospace and Electronic Systems Magazine, vol. 28 , no. 4, pp. 28-39, 2013.

[2] F. Colone, C. Bongioanni, and P. Lombardo, "Multifrequency integration in FM radio-based passive bistatic radar. Part II: direction of arrival estimation," IEEE Aerospace and Electronic Systems Magazine, vol. 28, no. 4, pp. 40-47, 2013.

[3] C. Shi, F. Wang, M. Sellathurai, and J. Zhou, "Transmitter subset selection in FM-based passive radar networks for joint target parameter estimation," IEEE Sensors Journal, vol. 16, no. 15, pp. 6043-6052, 2016.

[4] J. You, X. Wan, Y. Fu, and G. Fang, "Experimental study of polarisation technique on multi-FM-based passive radar," IET Radar, Sonar \& Navigation, vol. 9, no. 7, pp. 763-771, 2015.

[5] R. Raja Abdullah, N. Abdul Aziz, N. Abdul Rashid, A. Ahmad Salah, and F. Hashim, "Analysis on target detection and classification in LTE based passive forward scattering radar," Sensors, vol. 16, no. 10, p. 1607, 2016.

[6] H. Zeng, J. Chen, P. Wang, W. Yang, and W. Liu, "2-D coherent integration processing and detecting of aircrafts using GNSSbased passive radar," Remote Sensing, vol. 10, no. 7, p. 1164, 2018.

[7] L. Chen, P. Thevenon, G. Seco-Granados, O. Julien, and H. Kuusniemi, "Analysis on the TOA tracking with DVB-T signals for positioning," IEEE Transactions on Broadcasting, vol. 62, no. 4, pp. 957-961, 2016.

[8] T. Martelli, F. Colone, E. Tilli, and A. Di Lallo, "Multi-frequency target detection techniques for DVB-T based passive radar sensors," Sensors, vol. 16, no. 10, p. 1594, 2016.

[9] J. E. Palmer, H. Andrew Harms, S. J. Searle, and L. M. Davis, "DVB-T passive radar signal processing," IEEE Transactions on Signal Processing, vol. 61, no. 8, pp. 2116-2126, 2013.

[10] P. Hu, Q. Bao, and Z. Chen, "Weak target detection method of passive bistatic radar based on probability histogram," Mathematical Problems in Engineering, vol. 2018, Article ID 8243686, 10 pages, 2018.

[11] Y. Zheng and B. Chen, "Altitude measurement of low-angle target in complex terrain for very high-frequency radar," IET Radar, Sonar \& Navigation, vol. 9, no. 8, pp. 967-973, 2015.

[12] S. U. Pillai and B. H. Kwon, "Forward/backward spatial smoothing techniques for coherent signal identification," IEEE Transactions on Signal Processing, vol. 37, no. 1, pp. 8-15, 1989.

[13] B. Cai, Y. M. Li, and H. Y. Wang, "Forward/backward spatial reconstruction method for directions of arrival estimation of uncorrelated and coherent signals," IET Microwaves, Antennas \& Propagation, vol. 6, no. 13, pp. 1498-1505, 2012.

[14] A. Delis and G. Papadopoulos, "Enhanced forward/backward spatial filtering method for DOA estimation of narrowband coherent sources," IEE Proceedings Radar, Sonar and Navigation, vol. 143, no. 1, pp. 10-14, 1996. 
[15] K. Cui, W. Wu, J. Huang, X. Chen, and N. Yuan, "DOA estimation of LFM signals based on STFT and multiple invariance ESPRIT," AEÜ - International Journal of Electronics and Communications, vol. 77, pp. 10-17, 2017.

[16] E. M. Al-Ardi, R. M. Shubair, and M. E. Al-Mualla, "Computationally efficient high-resolution DOA estimation in multipath environment," IEEE Electronics Letters, vol. 40, no. 14, pp. 908910, 2004

[17] C. Qi, Y. Wang, Y. Zhang, and Y. Han, "Spatial difference smoothing for DOA estimation of coherent signals," IEEE Signal Processing Letters, vol. 12, no. 11, pp. 800-802, 2005.

[18] J. Shi, G. Hu, F. Sun, B. Zong, and X. Wang, "Improved spatial differencing scheme for 2-D DOA estimation of coherent signals with uniform rectangular arrays," Sensors, vol. 17, no. 9, p. 1956, 2017.

[19] Y. Wan, B. Xu, S. Tang et al., "DOA estimation for uncorrelated and coherent signals based on Fourth-order Cumulants," in Proceedings of the 2013 International Conference on Communications, Circuits and Systems, ICCCAS 2013, vol. 2, pp. 247-250, November 2013.

[20] X. Xu, Z. Ye, and J. Peng, "Method of direction-of-arrival estimation for uncorrelated, partially correlated and coherent sources," IET Microwaves, Antennas \& Propagation, vol. 1, no. 4, pp. 949-954, 2007.

[21] Y. F. Zhang and Z. F. Ye, "Efficient method of DOA estimation for uncorrected and coherent signals," IEEE Antennas and Wireless Propagation Letters, vol. 7, pp. 799-802, 2008.

[22] Z. Ye, Y. Zhang, and C. Liu, "Direction-of-arrival estimation for uncorrelated and coherent signals with fewer sensors," IET Microwaves, Antennas \& Propagation, vol. 3, no. 3, pp. 473-482, 2009.

[23] F. Liu, J. Wang, C. Sun, and R. Du, "Spatial differencing method for DOA estimation under the coexistence of both uncorrelated and coherent signals," IEEE Transactions on Antennas and Propagation, vol. 60, no. 4, pp. 2052-2062, 2012.

[24] L. Gan and X. Luo, "Direction-of-arrival estimation for uncorrelated and coherent signals in the presence of multipath propagation," IET Microwaves, Antennas \& Propagation, vol. 7, no. 9, pp. 746-753, 2013.

[25] H. Shi, W. Leng, A. Wang, and T. Guo, "DOA estimation for mixed uncorrelated and coherent sources in multipath environment," International Journal of Antennas and Propagation, vol. 2015, Article ID 636545, 8 pages, 2015.

[26] T. Yardibi, J. Li, P. Stoica, M. Xue, and A. B. Baggeroer, "Source localization and sensing: a nonparametric iterative adaptive approach based on weighted least squares," IEEE Transactions on Aerospace and Electronic Systems, vol. 46, no. 1, pp. 425-443, 2010.

[27] L. Du, T. Yardibi, J. Li, and P. Stoica, "Review of user parameterfree robust adaptive beamforming algorithms," Digital Signal Processing, vol. 19, no. 4, pp. 567-582, 2009.

[28] M. J. Jahromi and M. H. Kahaei, "Two-dimensional iterative adaptive approach for sparse matrix solution," IEEE Electronics Letters, vol. 50, no. 1, pp. 45-47, 2014.

[29] M. Barcelo, J. Lopez Vicario, and G. Seco-Granados, "A reduced complexity approach to IAA beamforming for efficient DOA estimation of coherent sources," EURASIP Journal on Advances in Signal Processing, vol. 2011, no. 1, pp. 1-16, 2010. 


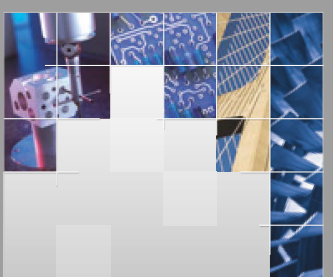

\section{Enfincering}
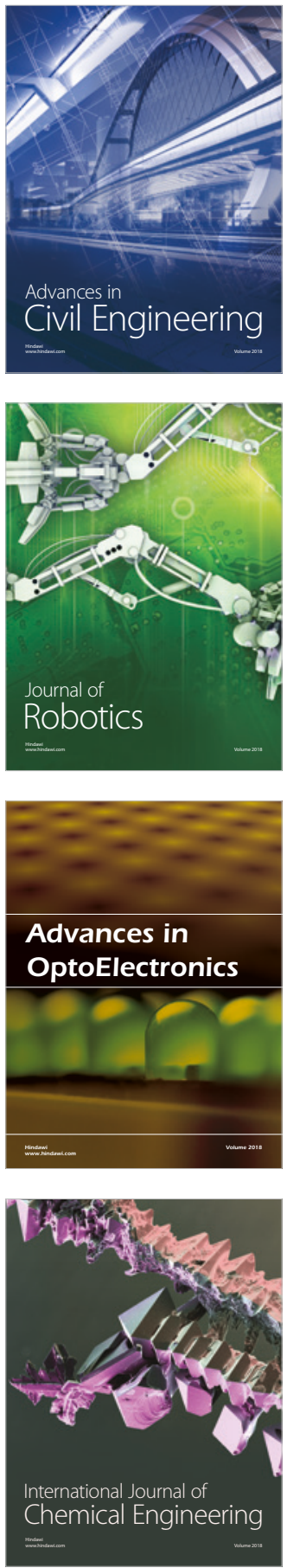

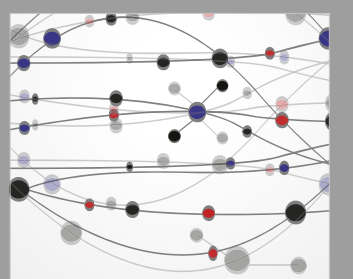

\section{Rotating \\ Machinery}

The Scientific World Journal

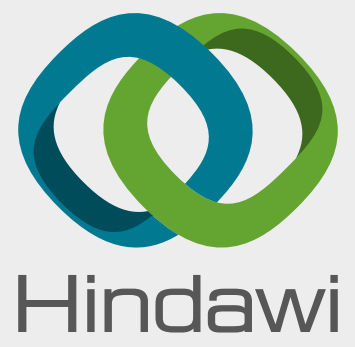

Submit your manuscripts at

www.hindawi.com
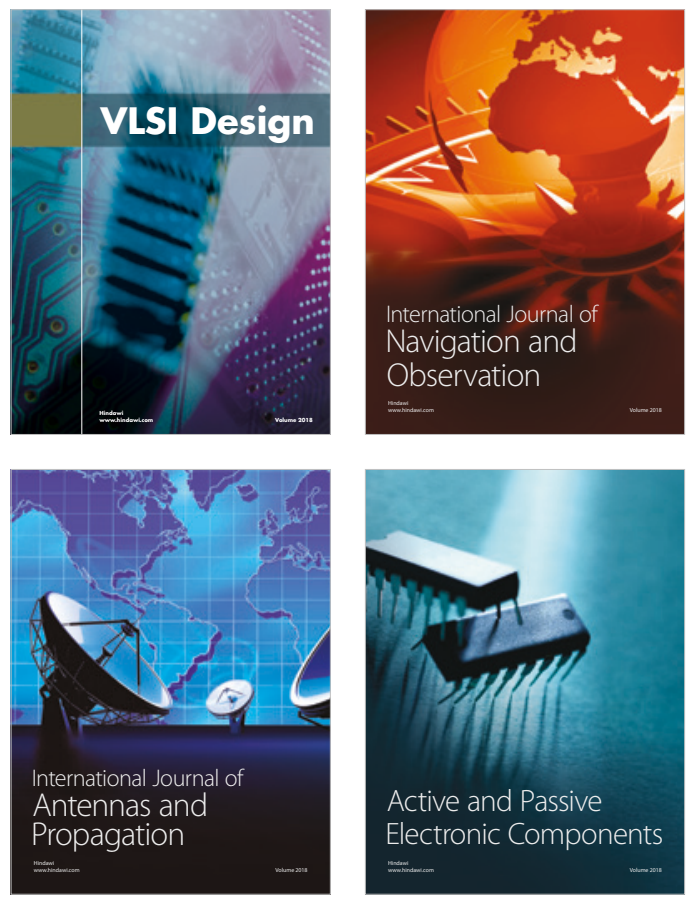
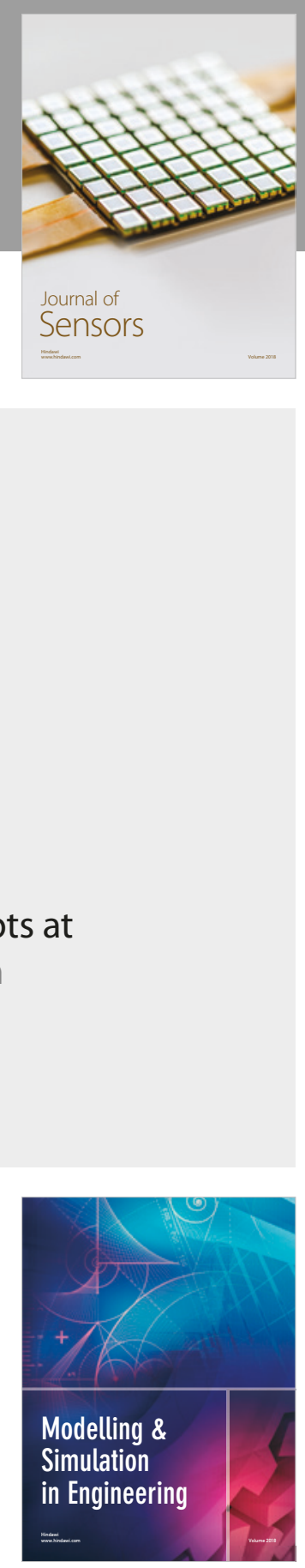

\section{Advances \\ Multimedia}
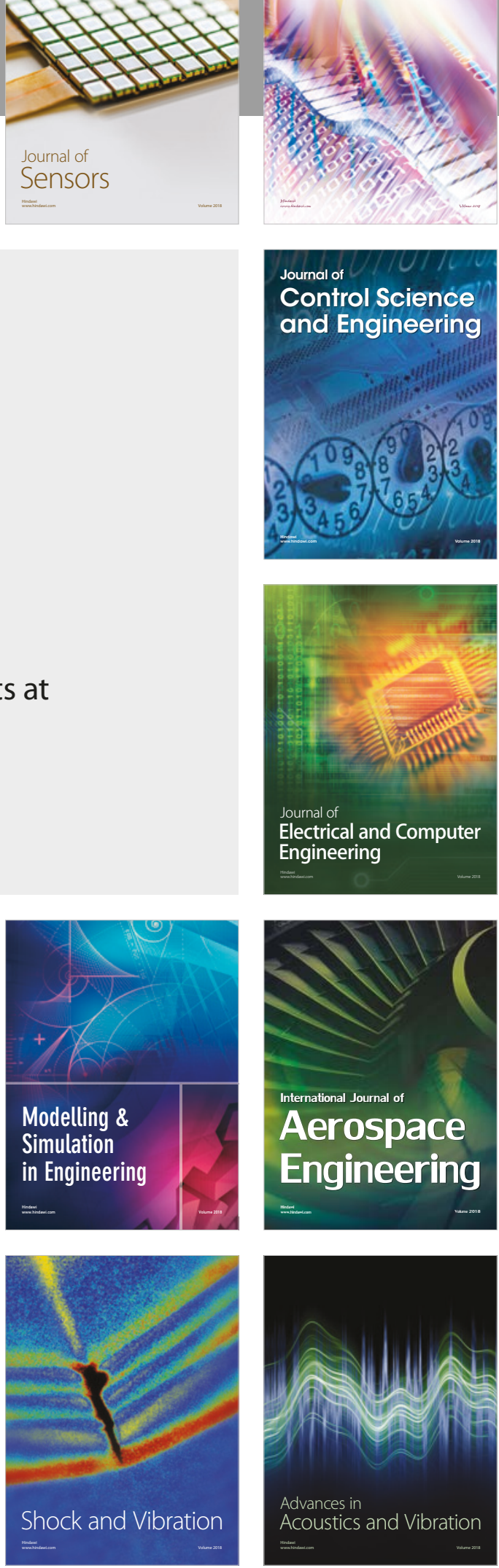